\title{
Influence of patients' emotional state on the recovery processes after a transient global amnesia
}

\author{
Audrey Noël ${ }^{1}$, Peggy Quinette ${ }^{1}$, Jacques Dayan ${ }^{1,2}$, Bérengère Guillery-Girard ${ }^{1}$, Pascale \\ Piolino $^{1,3}$, Alice Pélerin ${ }^{1,4}$, Vincent de la Sayette ${ }^{1,4}$, Fausto Viader $^{1,4}$, Béatrice Desgranges ${ }^{1}$ \\ and Francis Eustache ${ }^{1}$ \\ ${ }^{1}$ Inserm -EPHE-Université de Caen Basse - Normandie, Unité U923, Laboratoire de \\ Neuropsychologie, CHU Côte de Nacre, Caen, France. \\ ${ }^{2}$ Département de Psychiatrie de l'Enfant et de l'Adolescent, CHU Guillaume Régnier, \\ Rennes, France. \\ ${ }^{3}$ Laboratoire Psychologie et Neurosciences Cognitives, CNRS FRE 2927, Université \\ Paris Descartes, France. \\ ${ }^{4}$ Département de Neurologie, CHU Côte de Nacre, Caen, France.
}

Running title : Emotional and memory disorders after TGA

Correspondence to: Francis Eustache, Inserm-EPHE-Université de Caen Basse Normandie, Unité U923, Laboratoire de Neuropsychologie, CHU Côte de Nacre, 14033 Caen Cedex, France. Tel : + 332310651 97, Fax : + 332310651 98, e-mail : neuropsycho@chu-caen.fr 


\section{Abstract}

Introduction: Currently, there is no consensus on the delay necessary to a complete recovery after a transient global amnesia (TGA). However, it seems that slight episodic memory disorders extend beyond 24 hours. Although this impairment is probably a consequence of the TGA attack, other factors such as patients' emotional state can intervene in the slow recovery process.

Methods: In a first experiment, we studied the dynamic of recovery processes after a TGA. Thus, we assessed the anterograde and retrograde components of episodic memory in 19 patients one day, one month and one year after the attack. In a second experiment, we examined the impact of patients' emotional state on memory disorders, in using an original neuropsychological protocol (using material with emotional features) and an assessment of anxiety and depressive mood. This protocol was carried out in 19 other patients examined four months and one year after TGA.

Results: In the first experiment, we highlighted mild memory disorders affecting the anterograde component of episodic memory one day after the episode. In the second experiment, we showed these mild memory disorders could be detected several months after TGA. Moreover, patients who had the more depressive tendencies recognized the fewer items and those who displayed the highest level of anxiety supplied the fewer specific remote memories.

Conclusions: Our results showed that patients displayed very mild memory disorders several months after the episode of TGA, not affecting the daily routine. This impairment was influenced by patients' emotional state, which could suggest that a high level of anxiety or depression can slow down the recovery. However, we can not be sure that the deleterious effect of patients' emotional state on their cognitive performances is specific to TGA. Other investigations are necessary to unravel this issue. 
Keywords: transient global amnesia, follow-up study, episodic memory, anterograde memory, retrograde memoy. 


\section{Introduction}

Transient global amnesia (TGA) is a neurological syndrome characterized by a profound, time-limited memory impairment of acute onset, with no attendant neurological deficits. Many neuropsychological studies performed during the acute phase have reported that the impairment mainly consists of a massive disorder of episodic memory, resulting in both anterograde and retrograde amnesia. It is also generally acknowledged that after 4 to 6 hours, the memory impairment starts to recede (Hodges, 1998; Quinette et al., 2006). Furthermore, according to the criteria proposed by Hodges and Warlow (1990), an attack must be resolved within 24 hours.

However, an analysis of the literature reveals that slight memory disorders may persist after the episode. The majority of follow-up studies used a cross-sectional design: they examined a group of patients (between 14 and 55 individuals) just once after TGA, and to various delays. Moreover, within the same study, the delays between the follow-up assessment and the attack were very different according to each patient (Galassi et al., 1993; Le Pira et al., 2005; Neri et al., 1995). Thus, it is difficult to determine the time which is necessary to a full recovery. These studies highlighted a preservation of intellectual abilities (Borroni et al., 2004; Hodges and Oxbury, 1990; Mazzucchi et al., 1980) but showed that disorders of episodic memory persisted several months after the episode (Borroni et al., 2004; Hodges and Oxbury, 1990; Le Pira et al., 2005). After several years, studies reported discrepancies, showing sometimes large persistent memory disorders (affecting episodic and working memory; Borroni et al., 2004), sometimes only a slight residual deficit of episodic memory characterized by a recollection impairment (i.e. difficulties in retrieving additional contextual information about the learning event; Guillery-Girard et al., 2006), and sometimes no disorder (Uttner et al., 2007). Then, these data question the possibility of a full recovery after the episode. However, in some cases, the examination is performed a long time after the 
first attack and the deficits cannot necessarily be regarded as a consequence of TGA (but would be the result of pathology without links with TGA). Thus, taken together, these data emphasize the importance of adopting a longitudinal design with controlled time intervals between TGA and testing in order to better understand the recovery process and the long term prognosis of TGA.

Very few authors choose to use a longitudinal design, allowing assessing patients both during the episode and several times thereafter. Because this methodology is very demanding, these studies consisted predominantly in multiple case studies, and it makes difficult the generalisation of results. The most of these investigations highlighted persistent pathological performances in verbal and visual memory tasks up to 6 months after the episode (Caffara et al., 1981; Gallassi et al., 1986; Härting and Markowitsch, 1996; Quinette et al., 2003). Bartsch et al. (2006), however, did not find any episodic memory deficits in patients assessed 4-6 months later. Thus, in spite of a longitudinal design, these studies failed to determine the time necessary to a complete recovery, probably because the delay between the assessment and the attack was inadequate to observe the recovery process.

Thus, the aim of this study was to estimate the time necessary to a complete recovery of memory abilities. To this end, we followed two groups of patients several times after the episode in using comprehensive and original neuropsychological assessments. Because the most frequently reported disorders after the attack concern episodic memory, we carried out detailed neuropsychological examinations focused on the anterograde (including the assessment of autonoetic consciousness) and retrograde components of episodic memory. Moreover, we assessed metamemory abilities of patients in order to determine whether they were conscious of having residual memory disorders and whether they complained of these difficulties. The extent of the patients' memory disorders was gauged by making comparisons with a healthy control group. We also wanted to know what factors had an impact on the 
recovery processes. Thus, we studied the influence of the severity of TGA episode and of patients' emotional state on observed deficits. To the best of our knowledge, our prospective neuropsychological study is the first one to assess a group of TGA patients, with a longitudinal design.

\section{EXPERIMENT 1}

\section{1. Methods}

\subsubsection{Participants}

Nineteen patients ( 11 women and 8 men, with a mean age of 63.3 years, s.d. $=8$ and a mean educational level of 9.6 years, s.d. $=2.4$ ) were examined three time after the episode: around the next day (session 1), approximately one month later (session 2, mean=56.6 days afterwards, s.d.=21.3) and one year later (session 3, mean=13.9 months after, s.d.=2.4). In the majority of cases, the session 1 took place the morning following the episode (between 19 and 24 hours after the episode, except for 4 patients who were examined 7, 9, 34 and 111 hours after the end of TGA). In all cases, we made sure that patients had recovered their memory abilities i.e. that they were able to form new memories on the hours preceding the neuropsychological examination and to recall them.

By means of a codified procedure, senior neurologists made sure that all patients met the strict diagnostic criteria for TGA defined by Hodges and Warlow (1990). The mean duration of the episode was around 3 hours. The clinical and neurological examinations of the patients were all normal, except for memory disturbance. Brain CT scans and electroencephalograms performed were also normal. Clinical data of these 19 patients were already described in our previous review on TGA (Quinette et al., 2006), but the results reported here have never been published. All patients gave their informed consent to the 
study, which was conducted in line with the Declaration of Helsinki and approved by the local ethical committee.

For the neuropsychological investigations, the control group consisted of 10 women and 6 men, who were matched with the patients for age (mean age $=58.6$ years, s.d. $=8.4$; $\mathrm{t}(33)=1.66, \mathrm{p}=.11)$ and years of schooling $($ mean=11.5, s.d.=4; $\mathrm{t}(33)=-1.69, \mathrm{p}=.10)$. They did not have any neurological or psychiatric disorders. They underwent the same neuropsychological tests than patients three times (mean interval between the first and second sessions $=30.6$ days, s.d.=7.2, and between the first and third sessions $=12.1$ months, $s . d .=.3$ ).

\subsubsection{Neuropsychological assessment}

\subsubsection{General cognitive functions}

General cognitive functions were tested using the Mattis Dementia Rating Scale (Mattis, 1976) which comprises subtests of attention, initiation, construction, conceptualisation and memory. The total score and the scores for each subtest were calculated.

\subsubsection{Anterograde component of episodic memory}

Three tests were used to assess the anterograde component of episodic memory. They were chosen because they take into account the current definition of episodic memory, i.e. a memory system in charge of the encoding, storage and retrieval of personally experienced events, associated with a precise spatial and temporal context of encoding (Tulving, 1985, 2002): (i) memory for inter-item associations (What), (ii) spatial associations (Where) and (iii) temporal associations (When), coupled with an investigation of the type of consciousness 
(noetic or autonoetic) associated with recognition performances (Guillery-Girard et al., 2006). In the What, When and Where tasks, retrieval concerned either a target item associated with an other item, a target item associated with its location or associated with its order of presentation. The target (inter-item, spatial or temporal association) was systematically provided during the recognition phase of the three tasks, and patients then had to identify it, each time in choosing between two possible answers. The "What" task consisted in learning 12 pairs of words (for example: whistle - poster) which were displayed for 5 seconds. Word recognition was assessed by means of a dual forced-recognition task, in which only the second word of each pair was manipulated (e.g. whistle - poster and whistle - cotton), and associated with the Remember/Know paradigm (Gardiner et al., 2002), with an additional Guess response $(\mathrm{R} / \mathrm{K} / \mathrm{G})$. Patients had to give either a "Remember" response $(\mathrm{R})$, if retrieval was accompanied by the reliving of the context as a re-experiencing of the information from the learning context (i.e. thoughts, feelings or perceptions), or a "Know" response (K), if retrieval was achieved without any such access. The "Guess" response gave patients the possibility of signalling when they were not sure of their response. The "Where" task consisted in learning 12 words which were randomly distributed in the squares of a $6^{*} 6$ array. Patients had to learn the words and recognition was tested with a dual forced-recognition task, in which each of the 12 words was provided in the same grid, but in two different locations. Again, this was associated with the $\mathrm{R} / \mathrm{K} / \mathrm{G}$ paradigm. In the third task, i.e. the "When" task, patients had to learn two lists of 12 words. During the subsequent recognition task, patients were provided with word pairs featuring one word from the first list and one word from the second list. Patients were given instructions to point to the word belonging to the first list and to apply the $\mathrm{R} / \mathrm{K} / \mathrm{G}$ paradigm.

Thus, for each task, we obtained three scores of recognition (number of hits for "what", "where" and "when" tasks). As the two "Remember" and "Know" responses referred 
to independent processes, we determined a "Recollection" score and a "Familiarity" score according to Yonelinas' procedure. Although "Remember" score should provide a relative pure measure of recollection, "Know" score will not provide a pure measure of familiarity. Indeed, some of the items that elicit "Remember" responses are both recollected and familiar. Reporting the proportion of "Know" responses alone (as a measure of familiarity) underestimates the probability that an item is familiar. To determine the probability that an item is familiar, one must divide the proportion of "Know" responses by the opportunity the subject has to make a "Know" response (1-R) : F= K/(1-R) (Yonelinas, \& Jacoby, 1995). Thus, we obtained one score of "Recollection" and one score of "Familiarity" for each task ("what", "where" and "when").

Two different versions of this paradigm were devised and each one was alternately carried out by the subjects during the three sessions: the first version was used in sessions 1 and 3, while the second version was used in the second session. Even though the same version of the paradigm was used in session 1 and session 3, the practice effect was minimal, given that the interval between the two sessions was approximately one year. Furthermore, this anterograde episodic paradigm was part of a longer neuropsychological investigation, which further minimized the practice effect.

\subsubsection{Retrograde component of episodic memory}

An autobiographical fluency task was designed to assess the episodic component of remote autobiographical memory encompassing five life periods: 0-17 years, 18-30 years, more than 30 years except for the last 5 years, the last 5 years except for the last 12 months, and the last 12 months (Piolino et al., 2003). For each period, participants were given 2 minutes to retrieve the maximum number of single, specific, autobiographical events (episodic recall). If they failed to produce any recollections or produced only a general one, 
prompts were given, either helping them to find a recollection by providing cues about possible events (e.g. a birth, a marriage, a birthday, Christmas, etc.) or encouraging them to be more specific (e.g. describe what you did and felt, the circumstances, with whom, where and how it happened). The episodic features of the memories were tested after each assessed period by asking the participants to describe all the events in detail and then to indicate the nature of the subjective experience accompanying retrieval, by means of the Remember/Know procedure. The specificity of each recollection associated with a Remember judgment was measured by neuropsychologists using strict criteria based on an episodic scale which took into account the specificity of the content (single or repeated event), its spatiotemporal situation and the presence of details (perceptions, thoughts, feelings) (Piolino et al., 2003).

Episodic autobiographical memories were deemed to refer to a single event located in time and space, accompanied by phenomenological details (thoughts, emotions, images etc.) and associated with a Remember judgment. This procedure enabled us to distinguish strict episodic autobiographical memories fulfilling all the listed criteria from more general ones.

Two scores were collected for each time period: the total number of memories, whatever their nature, and the percentage of episodic memories.

\subsubsection{Memory complaints}

The scale we used is a French version of the Memory Functioning Questionnaire (Gilewski \& Zelinski, 1988; Israël \& Waintraub, 1997). Participants are asked to self-rate their memory performances in everyday life and quantify their memory complaints. The questionnaire consists of 60 items rated on a scale of 1 to 7 and provides 7 scores measuring the use of memory strategies, the frequency of forgetting, the seriousness attached to forgetting, memory of past events, retrospective functioning (i.e. estimation of current 
memory ability compared with past ability), the frequency of forgetting during reading and a general estimation of memory function. Higher scores reflect a more positive perception of memory function. A total score was obtained $/ 420(60 * 7)$. This questionnaire was administered once, during the second session.

\subsubsection{Statistical analysis}

In order to compare the patients' and controls' performances to each of the 3 sessions, we carried out a repeated-measures ANOVA, with group (normal controls, patients) as the between-subjects factor and session (first, second and third) as the within-subjects factor. When main effects of group and/or of session and/or interaction were found, supplementary post-hoc tests (PLSD Fisher) were carried out. For the memory complaints questionnaire, the performances of the two groups were compared by means of a one-way ANOVA. Main findings are described in the section below, while the scores and other analyses are set out in Table 1. Finally, we carried out correlations between the impaired cognitive scores and a clue of severity of episode (duration of TGA).

\subsection{Results}

\subsubsection{General cognitive functions}

There was a significant group effect for the overall score of the Mattis Dementia Rating Scale $(\mathrm{F}(1,33)=4.7, \mathrm{p}=0.03)$, with lower scores for patients (all sessions pooled). A significant main effect of session was also found $(\mathrm{F}(1,33)=5.6, \mathrm{p}<0.01)$. There was no significant interaction. The post hoc analyses showed that only patients significantly improved through the sessions. Indeed, the scores of session 1 were lower than those of 
session $2(\mathrm{p}<.01)$ and $3(\mathrm{p}<.01)$.

Detailed analyses performed for each subtest showed that main effects of group concerned the attention $(\mathrm{F}(1,33)=5.1, \mathrm{p}=0.03)$ and initiation subtests $(\mathrm{F}(1,33)=4.1, \mathrm{p}=0.05)$. Thus, patients' scores were lower than ones of the controls in both subtests (all sessions pooled). Moreover, a main session effect was also found for the attention subtest (F (1, 33 ) $=7.9, \mathrm{p}<0.001$ ), showing that the scores in session 1 were lower than in session 2 (in patients, $\mathrm{p}=.03$ ) and 3 (in patients and in controls, $\mathrm{p}<.01$ and $\mathrm{p}=.02$ respectively). There was also a session effect for the memory subtest $(\mathrm{F}(1,33)=3.7, \mathrm{p}=0.03)$. The scores obtained in session 1 were lower than those of session $2(\mathrm{p}=.05)$ and $3(\mathrm{p}=01)$, only in patients. There was no interaction effect.

\subsubsection{Anterograde component of episodic memory (Table 1)}

\subsubsection{1 "What" task}

First, there was only a significant effect of session for the recognition score (F (1, $33)=3.9, \mathrm{p}=0.02)$. Indeed, the performances in session 2 were lower than in session $3(\mathrm{p}<.01)$. Secondly, a main effect of group was revealed for the recollection score $(F(1,33)=6.1$, $\mathrm{p}=0.02$ ), indicating than patients' performances were lower than ones of controls in session 1 i.e. the day after the episode $(\mathrm{p}=.03)$. There was no session effect or interaction on Recollection score. Finally, no significant effect was found for the Familiarity score.

\subsubsection{2 "Where" task}

There was no significant result for the recognition score. On the contrary, we found a main group effect on the Recollection score $(\mathrm{F}(1,33)=5.0, \mathrm{p}=0.03)$. The most important difference between the scores of patients and controls concerned the session $1(p=.06)$. There was no session effect or interaction on Recollection score. No significant effect was found for 
the Familiarity score.

\subsubsection{3 "When" task}

A significant effect of session was revealed for the recognition score $(F(1,33)=3.8$, $\mathrm{p}=0.03$ ). A significant difference was observed between the sessions 1 and 3 in patients $(\mathrm{p}=.02)$ and between the sessions 2 and 3 in controls $(\mathrm{p}=.04)$. There was no other significant result for the recognition score. An analysis of the Remember/Know performances showed only a main effect of session on the Recollection score $(F(1,33)=17.5, p<0.001)$ without significant group effect or interaction. In both patients and controls, the scores in session 3 were higher than in sessions $1(\mathrm{p}=.004$ and $\mathrm{p}<.001$, respectively) and $2(\mathrm{p}=.05$ and $\mathrm{p}<.001$, respectively). No significant main effect or interaction was found for the Familiarity score.

\subsubsection{Retrograde component of episodic memory (Table 1)}

Repeated-measures ANOVAs (Group x Session) were performed on both scores (i.e. total number of recalled memories and percentage of episodic memories) for each period. There were no significant effects of group, session or interaction for the number of responses for each period, except for an interaction for the most recent one (last 12 months) (F (1, $32)=4.89, \mathrm{p}=.01$ ). A post-hoc analysis revealed that patients produced fewer autobiographical memories than controls, one year after the episode $(\mathrm{p}=.03)$. Moreover, patients' performances improved between the first and the second sessions ( $\mathrm{p}=.04)$ and lightly decreased to the third session. The profile of controls' performances was opposed to the one of patients. The controls' results were lower in session 2 than session $1(\mathrm{p}=.08)$. The number of recalled memories in session 3 were significantly higher than one of session $2(\mathrm{p}=.003)$.

Concerning the percentage of episodic memories, no significant effect of group, session or interaction was found, whatever the period. 


\subsubsection{Memory complaints}

A one-way ANOVA showed that patients did not have more memory complaints than controls. This analysis was carried out on both the subtest scores and the total score (patients' mean total score $=261.9$, s.d. $=34.4$; controls' mean total score $=264.2$, s.d. $=32.9$ ).

\subsubsection{Impact of the severity of episode on recovery processes}

We found negative correlations between the duration of episode and the scores of the Mattis $(\mathrm{r}=-.62 ; \mathrm{p}<.01)$, initiation $(\mathrm{r}=-.65 ; \mathrm{p}<.01)$ and memory $(\mathrm{r}=-.53 ; \mathrm{p}<.03)$ subtests obtained during the first session. The recollection score of "where" task (recognition of location) in session 1 was also negatively correlated with the duration of the episode $(r=-.64 ; p<.01)$.

\subsection{Comments}

To sum up, TGA patients displayed very mild neuropsychological deficits during the follow-up period. The analysis of results to the Mattis scale showed that patients had difficulties in initiation, attention and memory subtests during the first session i.e only the next day after the episode. These disorders were more extended in patients who had suffered from the longest TGA episodes. The more detailed assessments of the anterograde component of episodic memory allowed emphasizing a deficit of the Recollection processes in two of accomplished tasks (what and where tasks). Thus, patients did not achieve to perfectly encode the information itself (what task) and the location of information (where task). The more the TGA episode had been severe, the more patients' performances were weak in the task of location recognition. These disorders were also limited to the first session. On the contrary, the temporal information was well treated by patients. Concerning the retrograde component 
of episodic memory, we found that patients produced fewer recent memories (for the 12 last months) than controls during the third session i.e. one year after the episode.

With regard to these results, we asked us if the tests used in this first experiment were sensitive enough to detect very mild disorders after the episode. Thus, instead of a multiple choice recognition task comprising only two possible responses (one hit and one filler), we carried out in our second experiment a yes/no recognition task in which we presented only one item at the screen and participants had to determine if it belonged to the learnt list. Regarding the retrograde component, in the first experiment, we tested the access and the nature of recalled memories $(\mathrm{R} / \mathrm{K}$ paradigm). In our second experiment, we focused on an assessment of the quality of recalled episodic memories. Moreover, in a previous study (Noël et al., 2008), we showed that TGA was associated to an increasing of anxiety and depression level during the acute phase. This change of patients' emotional state had a deleterious effect on cognitive performances of patients. Thus, we wanted to also determine whether patients still displayed a high level anxiety and depression after the TGA episode and whether this one interfered with or even slow down the recovery process after TGA. To gain a better understanding of this putative interaction, we investigated the mood congruency effect (in using a materiel with emotional features). Finally, we also took account of the severity of TGA episode in order to determine its influence in the recovery processes.

\section{EXPERIMENT 2}

\section{1 Methods}

\subsubsection{Participants}

Nineteen new patients ( 11 women and 8 men with a mean age of 61 years, s.d.=7.3, and a mean education level of 10.15 , s.d.=3.6) were examined. These 19 patients were 
different of those described in the first experiment. They were all examined approximately four months after a TGA episode (session $1, \mathrm{~m}=4.3$ months, s.d. = 1.9) and ten of these ones ( 4 women and 6 men with a mean age of 60.8 years, s.d.=7, and a mean education level of 11.4 years, s.d.=3.9) were reassessed approximately one year after the attack (session $2, \mathrm{~m}=$ 15.1 months, s.d. $=1.7$ ). All patients were recruited with the same strict recruitment methods described in the first experiment. These 19 patients belonged to an initial cohort of thirty-five TGA patients examined during the acute phase (Noël et al., 2008) on a period of 3 years. The 19 patients that we followed up in the present study were ones that we met in first during the first and second years and then that we contacted again four months and one year later. We reported here these original data.

The same assessments were conducted on a control group of 26 subjects (session 1, 12 women and 14 men, mean age $=60.2$, s.d. $=7.3$ and mean education level $=11.8$, s.d. $=3.7$ ) already described in Noël et al. (2008). Ten of these twenty-six controls (3 women and 7 men with a mean age of 64.4 years, s.d.=3.9, and a mean education level of 11.9 years, s.d.=3.7) were reassessed twelve months later.

\subsubsection{Neuropsychological assessment}

\subsubsection{General cognitive functions}

General cognitive functions were tested using the Mattis Dementia Rating Scale (Mattis, 1976) which comprises subtests of attention, initiation, construction, conceptualisation and memory. The total score and the scores for each subtest were calculated.

\subsubsection{Anterograde component of episodic memory}


The anterograde component of episodic memory was assessed by means of an yes/no recognition task comprising emotional words. During the study session, participants had to memorize a list of 10 positive, 10 negative and 10 neutral words (intentional encoding) and judge the emotional valence of these 30 words on an 8-point scale (from 1=highly negative to $8=$ highly positive). The words were displayed one by one on a screen of computer in pseudorandom order. To scroll the words, the participants had to press one of 8 buttons corresponding to the valence of item. The test session took place 5 minutes later. One score of recognition was obtained for each valence list (accuracy score for positive, negative and neutral items). This score was calculated according to the Gardiner's procedure $\left(\mathrm{A}^{\prime}=1 / 2+\right.$ [[(Hit - FA $)(1+$ Hit -FA $)] /$ 4Hit $(1-$ FA $)] ;$ FA = false alarm; Gardiner et al., 2002). To assess the state of consciousness associated with each response, we used the Remember/Know/Guess (R/K/G) paradigm (Gardiner et al., 2002). Two scores (Recollection and Familiarity scores) were calculated for each valence according to the number of Remember (R) and Know (K) responses (for more details, see experiment 1). We used a parallel version of this task for the second session.

\subsubsection{Retrograde component of episodic memory}

The autobiographical memory task, derived from Piolino et al.'s semi-structured questionnaire (TEMPau task, Piolino et al., 2003), allowed us to gauge subjects' ability to recall specific, detailed events situated in time and space from three lifetime periods (18-30 years old, last 10 years except the last 12 months, and last 12 months). The number of life periods was reduced in comparison of the fluency autobiographical task described in the first experiment, as we asked to patients to tell exhaustively two memories of their life for each period (and not only to list events). We gave to participants a very precise definition of a specific event, that is, a unique event lasting less than a day, located in time and space, which 
can be recalled with sensory, perceptual of affective details. Two specific memories were requested for each lifetime period (randomly displayed on a screen of computer). The quality of each episodic memory (specificity score) was scored on 4 points: one point if the event recalled was unique (event being produced only once), 1 point if the event lasted less than 24 hours, 1 point if it was located in time and space and 1 point if it comprises at least two specific details (belonging exclusively to this event). We obtained one specificity score for each period in working out the average of two memories produced for each lifetime period (3 specificity scores). After each recall, three Likert scales (in 6-point) assessing respectively the state of consciousness (from 1 for a simple feeling of familiarity up to 6 for an impression of re-experiencing the past event), the emotional intensity (from 1 for a low intensity up to 6 for an high intensity) and the valence of each memory (from 1 for unpleasant event up to 6 for a pleasant event) were fulfilled by patients. We calculated one score of Recollection, one score of emotional intensity and one score of emotional valence for each period in working out the average of responses on the Likert scales for the two memories produced by lifetime period (3 Recollection scores, 3 emotional intensity scores and 3 emotional valence scores).

\subsubsection{Psychopathological assessments}

Two questionnaires assessed the presence of anxiety on the basis of the State-Trait Anxiety Inventory (STAI, Spielberger, 1983). We assessed mood over the two weeks prior to the neuropsychological examination using the abridged version of the Beck Depression Inventory, or BDI (Beck et al., 1974), and at the actual time of the examination using the Adjective Mood Scale, or Bf-S ("Befindlichkeits-Skala", Von Zerssen et al., 1970). The higher the scores on this scale, the more depressed the patients were assumed to be, at the time of examination. 


\subsubsection{Statistical analysis}

The neuropsychological and psychopathological data were analyzed using repeatedmeasures ANOVA, with group (normal controls, patients) as a between-subjects factor and session (first and second sessions) as the within-subjects factor. When we noticed a significant group, session or interaction effects, we carried out post-hoc analyses (PLSD Fisher test) to specify these effects.

We then carried out correlations between the patients' impaired memory scores, the data obtained by means of anxiety and mood scales and a clue of severity of TGA episode (duration of the episode) in order to determine the influence of these factors on the memory disorders observed after the episode. To increase the statistical power, we reduced the number of cognitive variables, retaining only the most impaired cognitive scores on each task (anterograde and retrograde components of episodic memory, Table 2). The same correlations were carried out in controls, in order to determine whether the psychopathological factors had a deleterious effect on memory in all subjects or whether this effect affected only vulnerable subjects as TGA patients who present memory disorders. According to the number of subjects in each group included in the analysis, we used either Spearman's or Bravais-Pearson's correlation coefficients. A probability level of 0.05 was adopted for all analyses.

\subsection{Results}

\subsubsection{Neuropsychological assessments after TGA}

\subsubsection{General cognitive functions}

There was no significant difference between patients and controls on the total score of Mattis scale or on the scores of subtests.

\subsubsection{Anterograde component of episodic memory (Table 2)}


The analysis of the accuracy scores for each emotional valence failed to reveal any significant effect of group, session or interaction on the scores for positive and negative items. There was, however, a significant group effect on the accuracy scores for neutral items $(\mathrm{F}(1,18)=4.9 ; \mathrm{p}=.04)$. Patients recognized fewer neutral words than controls on the two sessions together. There was no significant session or interaction effect for neutral items. Furthermore, there was no significant difference between patients and controls on the recollection and familiarity scores, whatever the valence and session.

\subsubsection{Retrograde component of episodic memory (Table 2)}

First, we conducted an analysis of the specificity scores for each life period. We found no significant effect of group, session or interaction on the scores for the " $18-30$ years old" and "last 12 months" periods. Concerning the "last 10 years" period, there was a significant effect of group $(\mathrm{F}(1,18)=10.8 ; \mathrm{p}<.01)$ and interaction $(\mathrm{F}(1,18)=8.8 ; \mathrm{p}<.01)$, but no effect of session. The post-hoc analyses revealed that patients supplied fewer specific memories than controls, but only in the first session (four months after the episode, $\mathrm{p}=.04$ ).

As far as the recollection score is concerned, analyses failed to reveal any significant effect of group, session or interaction for the "18-30 years old" period. There was, however, a significant effect of group on this score for the "last 10 years" period $(\mathrm{F}(1,18)=5.1, \mathrm{p}=.04)$. Thus, patients assessed their memories as being less episodic than controls, on the two sessions together. No effect of session or interaction was found for the "last 10 years" period. Our analyses also showed a significant interaction effect for the last period, i.e. "the last 12 months" $(F(1,18)=4.8, \mathrm{p}<.05)$. There was no effect of group or session, although a marginal effect $(\mathrm{p}<.08)$ was observed on the recollection score, during the second session, as patients assessed their most recent memories as being less specific than controls.

There was no significant effect of group, session or interaction on the intensity scores, 
whatever the period.

Similarly, no significant effect of group, session or interaction on the valence score was found for the "18-30 years old" period, although we did highlight a group effect on the valence scores for the two last periods, i.e. "the last 10 years" $(F(1,18)=6.6, p=.02)$ and "the last 12 months" $(\mathrm{F}(1,18)=10.8, \mathrm{p}<.01)$. This result means that, whatever the session, patients supplied more negative memories than controls for these two lifetime periods. Effects of session and interaction were not significant.

In sum up, the disorders affecting the recall of autobiographical memories concerned specifically the "last 10 years" period.

\subsubsection{Influence of patients' emotional state on memory performances}

We began by comparing the scores of the patients and controls on the psychopathological scales and found that there was no difference in the levels of anxiety and depression measured on the four psychopathological scales, whatever the session (Table 3).

We only report the significant correlations between the psychopathological scores and impaired memory scores of patients and controls. As far as the anterograde component of episodic memory is concerned, the recognition score for neutral items was negatively with the score obtained to Adjective Mood Scale ( $\mathrm{r}=-.49 ; \mathrm{p}=.03$ ), during the first session (four months after the episode). Thus, the more the mood of patients was negative at the time of the episode (current depressive mood), the less patients recognized neutral items. Regarding the retrograde component of episodic memory, the specificity score for the second period ("the last 10 years") was negatively correlated with the anxiety state score $(r=-.71 ; \mathrm{p}<.02)$, during the first session (four months after the episode). In other word, the more their level of anxiety at the time of the examination was high, the less their memories for the intermediate "last 10 years" period was specific. No other correlation was significant. 
There was no significant correlation between the severity of TGA episode and the impaired memory scores.

In controls, we only observed two significant correlations. Thus, the specificity score for the second period ("the last 10 years") was negatively correlated with the anxiety trait score $(\mathrm{r}=-.40, \mathrm{p}=.05)$ and the BDI depression score $(\mathrm{r}=-.49, \mathrm{p}=.01)$.

Lastly, we carried out correlations between the patients' age, their level of education and the scores they obtained on the memory tasks. We found no deleterious effect of age or level of education on memory performances.

\subsection{Comments}

First, we showed the presence of very mild memory deficit several months after the episode. Indeed, concerning the anterograde component of episodic memory, we found that patients had lower performances than controls. However, this difference was very weak and was significant exclusively when the two sessions were pooled. Moreover, patients produced less episodic memories than controls during the first session (about 4 months after the episode) but only for the intermediate lifetime period i.e. the 10 last years. This result was confirmed by a weak feeling of recollection for this lifetime period. Secondly, we highlighted that the level of patients' anxiety and depression were became normal again after the episode. Finally, our results emphasize the level anxiety and depression influenced the memory performances. Indeed, patients who presented the highest anxiety and depression levels displayed the most impaired cognitive profile.

\section{General discussion}

The first aim of our study was to determine whether TGA patients kept sequels of the episode, several months after this one. Our results showed that TGA patients displayed only 
very mild neuropsychological disorders after the episode. Indeed, general cognitive functions, assessed by means of the Mattis scale, were impaired only the following day after the episode. More precisely, the difficulties were observed for attention, initiation and memory subtests. Other studies (Gallassi et al., 1986; Hodges and Ward, 1989) highlighted similar deficits in the days following the episode. Then, the recovery appeared to be very fast.

Concerning the anterograde component of episodic memory, we also showed a mild impairment. In the first experiment, only a deficit of autonoetic consciousness was highlighted the following day after the episode. In a previous follow-up study, GuilleryGirard et al. (2006) already mentioned that patients preferentially used familiarity (Know responses) rather than re-living (Remember responses) processes. These authors claimed that patients had an inability to re-experience the original laboratory event i.e. to retrieve additional information about the learning event. It has been proposed that recognition memory relies on two independent retrieval processes: a familiarity and a recollection process (Yonelinas, 2002). TGA patients would use rather a familiarity process than recollection to perform the recognition task. This deficit was limited to tasks which implicated to memorize the content of information and its location but not its temporal order. The two tasks requiring the association of words pairs and the association of word-location, respectively, likely involve medial temporal lobes (Hayes et al 2004; Jackson and Schacter, 2004). On the contrary, the "when" task which consists in a temporal judgement (memorize the order of words in a list) would rely on the bilateral prefrontal regions (Suzuki et al., 2002). Interestingly, recent imaging studies (Bartsch et al., 2006; Lee et al., 2007; GonzalesMartinez et al., 2010) carried out in TGA showed focal lesions of the hippocampus and more particularly of CA-1 neurons. Moreover, the size of lesions was correlated with the deficit of performance in a place memory task (Bartsch et al., 2010). In our second experiment, we found a mild memory impairment several months after the episode. These disorders were 
restricted to one category of items: the neutral ones. Thus, patients seemed to benefit from the emotional valence of the stimuli and were thus able to reach normal performance levels. It is now widely acknowledged that emotion enhances memory abilities (Kensinger, Garoff-Eaton and Schacter, 2008) but two hypotheses have been put forward to explain this effect. First, it was postulated that emotional stimuli are more distinctive than neutral (Christianson et al., 1991; Ochsner, 2000). Secondly, emotion can serve as an unifying theme to memories, allowing items to be more easily clustered (Kensinger and Corkin, 2004). Although no significant difference was found between the Recollection scores of the two groups, we did notice an improvement in patients' scores between the first and second sessions, particularly for neutral items.

Regarding the retrograde component of episodic memory, in the experiment 1 , we found that patients produced less autobiographical memories than controls for the last lifetime period (12 last months), only one year after the episode. Whereas the total number of memories recalled by patients was stable across sessions, this one of controls decreased in session 2 and considerably increased in the session 3. We think that these fluctuations of performances are probably due to a weariness effect or a loss of motivation in controls who participated to this experiment voluntarily and benevolently, contrary to patients who found a benefit in these assessments (notably reassurance). Thus, according to us, the differences observed between patients and controls reflect rather a motivation effect than a genuine deficit of access to autobiographical memories. However, a default of retrieval strategies in memory had already been shown in TGA patients, notably using verbal fluency tasks (Kessler et al., 2001). In experiment 2, we choose to assess the quality of autobiographical memories produced by patients. Our results showed that memories evoked by patients were less episodic than ones of controls for the intermediate period (the "last 10 years"), only for the first session, i.e. some months after the episode. Moreover, patients correctly judged themselves 
that the memories produced for this period were less specific, as showed by their assessment on the Recollection scale. The retrieval of memories for this lifetime period was probably more difficult than for other periods, given that for the most recent period ("last 12 months"), patients and controls alike tended to recall events that had occurred during the week preceding the neuropsychological examination. As Conway (1990) emphasises, recent events, or "fresh memories", often contain specific details because they continue to have a direct impact on people's current lives. The "18-30 years" period also has a particular resonance and corresponds to the reminiscence bump, which is the richest lifetime period in terms of selfrelevant experiences. It is characterized by extremely numerous, detailed, intense and selfrelevant memories, for example about one's wedding, the birth of children or one's first job (Berntsen and Rubin, 2002), which facilitate recall. The intermediate period does not allow any such strategy and does not have any particular characteristics.

The second aim of our study consisted to gain a better understanding of factors influencing the recovery process after the TGA episode. In the one hand, we wanted to determine the impact of the severity of episode on disorders observed after TGA. Thus, we showed that the duration of episode was negatively correlated with impaired scores but only in the hours following the episode.

In the other hand, we were particularly interested in the role played by anxiety and depressed mood in the recovery processes. Indeed, during the acute phase, we found that memory disorders were associated with a sudden downward swing in the patients' emotional state (increased level of anxiety and onset of depressive symptoms). Like the memory disorders, these psychopathological disorders ebbed away during the recovery phase (Noël et al., 2008), although they could still be detected in some patients a day after the episode and were severe enough to affect memory performances (Noël et al., 2007). Several months after 
the episode, patients did not show psychopathological disorders anymore. However, patients who presented the highest anxiety and depression levels at the time of the examination (current emotional state) displayed the most severely impaired cognitive profile (affecting both anterograde and retrograde components of episodic memory). In controls, it is a stable and prolonged emotional state (trait anxiety and depression) that influenced memory capacities. In spite of this major difference between patients and controls, it is difficult to know if the influence of patients' emotional state on the performances in episodic memory is specific to TGA. The inclusion of another control group comprising patients who lived an acute episode such as a transitory ischemic accident could allow us responding to this question.

In conclusion, TGA patients exhibited only very mild neuropsychological disorders after the episode, not affecting their daily routine. Moreover, they did not report memory complaints. However, these patients informed us of one preoccupation which concerned the putative outbreak of a new episode of TGA. Thus, we suggest that these patients consult a neurologist about one month after the episode in order to be reassured on a putative new TGA episode, given the risk is weak.

\section{Acknowledgments}

This study was funded by Caen University Hospital as part of a clinical research project and by the Servier laboratory. The authors would like to thank Philippe Conejero for his editorial work, Elizabeth Wiles-Portier for reviewing the English style, Dr Sophie Marquis and the staff of the emergency unit of Caen University Hospital for their invaluable collaboration, and the psychologists Emilie Morançais, Elisa Roger, David Déprès and Aurelija Juskenaïte for their help in collecting the data. 


\section{References}

Bartsch T, Alfke K, Stingele R, Rohr A, Freitag-Wolf S, Jansen O, and Deuschl G. Selective affection of hippocampal CA-1 neurons in patients with transient global amnesia without long-term sequelae. Brain, 129: 2874-84, 2006.

Bartsch T, Schönfeld R, Müller FJ, Alfke K, Leplow B, Aldenhoff J, Deuschl G, and Koch JM. Focal lesions of human hippocampal CA1 neurons in transient global amnesia impair place memory. Science, 328: 1412-1415, 2010.

Beck AT, Rial WY, and Rickets K. Short form of Depression Inventory: cross validation. Psychological Reports, 34: 1184-6, 1974.

Berntsen D and Rubin DC. Emotionally charged autobiographical memories across the life span: the recall of happy, sad, traumatic, and involuntary memories. Psychology and Aging, 17: 636-52, 2002.

Borroni B, Agosti C, Brambilla C, Vergani V, Cottini E, Akkawi N, and Padovani A. Is transient global amnesia a risk factor for amnestic mild cognitive impairment? Journal of Neurology, 25: 1125-1127, 2004.

Bradley MM, Greenwald MK, Petry MC, and Lang PJ. Remembering pictures: pleasure and arousal in memory. Journal of Experimental Psychology. Learning Memory and Cognition, 18: 379-90, 1992.

Caffarra P, Moretti G, Mazzucchi A, and Parma M. Neuropsychological testing during a transient global amnesia episode and its follow-up. Acta Neurologica Scandinavica, 63: 44-50, 1981.

Christianson SA, Loftus EF, Hoffman H, and Loftus GR. Eye fixations and memory for emotional events. Journal of Experimental Psychology: Learning, Memory and Cognition, 17: 693-701, 1991. 
Conway MA. Autobiographical Memory: An introduction. Philadelphia: Open University Press, 1990.

Eustache F, Desgranges B, Laville P, Guillery B, Lalevée C, Schaeffer S, de la Sayette V, Iglesias S, Baron JC, and Viader F. Episodic memory in transient global amnesia: encoding, storage, or retrieval deficit? Journal of Neurology, Neurosurgery and Psychiatry, 66: 148-154, 1999.

Gallassi R, Lorusso S, and Stracciari A. Neuropsychological findings during a transient global amnesia attack and its follow-up. Italian Journal of Neurological Sciences, 7: 45-49, 1986.

Gallassi R, Stracciari A, Morreale A, Lorusso S, Rebucci GG, and Lugaresi E. Transient global amnesia: neuropsychological findings after single and multiple attacks. European Neurology, 33: 294-298, 1993.

Gardiner JM., Ramponi C, and Richardson-Klavehn A. Recognition memory and decision processes: a meta-analysis of remember, know, and guess responses. Memory 10: 83-98, 2002.

Gilewski MJ and Zelinski EM. Questionnaire assessment of memory complaints. In Poon LW (Ed), The Handbook of clinical memory assessment of older adults. Washington: American Psychological Association, 1988: 93-107.

Gonzalez-Martinez V, Comte F, de Verbizier D, Carlander B. Transient global amnesia: concordant hippocampal abnormalities on positron emission tomography and magnetic resonance imaging. Archives of Neurology, 67:510, 2010.

Guillery-Girard B, Desgranges B, Urban C, Piolino P, de la Sayette V, and Eustache F. The dynamic time course of memory recovery in transient global amnesia. Journal of Neurology, Neurosurgery and Psychiatry, 75: 1532-1540, 2004.

Guillery-Girard B, Quinette P, Desgranges B, Piolino P, Viader F, de la Sayette V, and 
Eustache F. Long term memory following transient global amnesia: an investigation of episodic and semantic memory. Acta Neurologica Scandinavica, 114: 329-333, 2006.

Härting C and Markowitsch HJ. Different degrees of impairment in anterograde/retrograde memory and recall/recognition performance in a transient global amnesic case. Neurocase, 2: 45-49, 1996.

Hayes SM, Ryan L, Schnyer DM, and Nadel L. An fMRI study of episodic memory: retrieval of object, spatial and temporal information. Behavioral Neuroscience, 118: 885-896, 2004.

Hodges JR. Unraveling the enigma of transient global amnesia. Annals of Neurology, 43: 151$153,1998$.

Hodges JR and Oxbury SM. Persistent memory impairment following transient global amnesia. Journal of Clinical and Experimental Neuropsychology, 12: 904-920, 1990.

Hodges JR and Ward CD. Observations during transient global amnesia: a behavioural and neuropsychological study of five cases. Brain, 112: 595-620, 1989.

Hodges JR and Warlow CP. Syndromes of transient amnesia: towards a classification. A study of 153 cases. Journal of Neurology, Neurosurgery and Psychiatry, 53: 834-843, 1990.

Israël L and Waintraub L. Questionnaire du fonctionnement de la mémoire. Auto-évaluation des plaintes mnésiques. In Guelfi JD (Ed), L'évaluation clinique standardisée en psychiatrie. Paris: Editions Médicales Pierre Fabre, 1997: 493-497.

Jackson O, and Schacter DL. Encoding activity in anterior medial temporal lobe supports subsequent associative recognition. Neuroimage, 21: 456-462, 2004.

Jäger T, Szabo K, Griebe M, Bäzner H, Möller J and Hennerici MG. Selective disruption of hippocampus-mediated recognition memory processes after episodes of transient global amnesia. Neuropsychologia, 47: 70-76, 2009. 
Kensinger EA, and Corkin S. Memory enhancement for emotional words: Are emotional words more vividly remembered than neutral words? Memory and Cognition, 31: 11691180, 2003.

Kensinger EA, Garoff-Eaton RJ, and Schacter DL. Effects of emotion on memory specificity in young and older adults. Journal of Gerontology: Psychological Science, 62: 208-215, 2007.

Kessler J, Markowitsch HJ, Rudolf J, and Heiss WD. Continuing cognitive impairment after isolated transient global amnesia. The International Journal of Neuroscience, 106: 159$168,2001$.

Lee HY, Kim JH, Weon YC, Lee JS, Kim SY, Youn SW and Kim SH. Diffusion-weighted imaging in transient global amnesia exposes the CA1 region of the hippocampus. Neuroradiology, 49: 481-487, 2007.

Le Pira F, Giuffrida S, Maci T, Reggio E, Zappala G, and Perciavalle V. Cognitive findings after transient global amnesia: role of prefrontal cortex. Applied Neuropsychology, 12: 212-217, 2005.

Lucas JA, Ivnik RJ, Smith GE, Bohac DL, Tangalos EG, Kokmen E, Graff-Radford NR, and Petersen RC. Normative data for the Mattis Dementia Rating Scale. Journal of Clinical and Experimental Neuropsychology, 20: 536-547, 1998.

Mattis S. Mental status examination for organic mental syndrome in the elderly patient. In Bellack L, and Karasu TB (Eds), Geriatric psychiatry: a handbook for psychiatrics and primary care physicians. New York: Grune and Stratton, 1976: 77-121.

Mazzucchi A, Moretti G, Caffarra P, and Parma M. Neuropsychological functions in the follow-up of transient global amnesia. Brain, 103: 161-178, 1980.

Nakada T, Kwee IL, Fujii Y, and Knight RT. High-field, T2 reversed MRI of the hippocampus in transient global amnesia. Neurology, 64: 1170-1174, 2005. 
Nausieda PA and Sherman IV. Long-term prognosis in transient global amnesia. JAMA: the Journal of the American Medical Association, 241: 392-393, 1979.

Neri M, Andermarcher E, De Vreese LP, Rubichi S, Sacchet C, and Cipolli C. Transient global amnesia: memory and metamemory. Aging, 7: 423-429, 1995.

Noël A, Quinette P, Guillery-Girard B, Dayan J, Katis S, Piolino P, Abadie P, de la Sayette V, Marquis S, Viader F, Desgranges B, and Eustache F. How psychopathological factors affect both the onset of and recovery from transient global amnesia. Psychological Medicine, 20: 1-4, 2007.

Noël A, Quinette P, Guillery-Girard B, Dayan J, Piolino P, Marquis S, de la Sayette V, Viader F, Desgranges B, and Eustache F. How do psychopathological factors and memory disorders interact during transient global amnesia? British journal of psychiatry, 193: 145-151, 2008.

Ochsner KN. Are affective events richly "remembered" or simply familiar? The experience and process of recognizing feelings past. Journal of Experimental Psychology: General, 129:242-261, 2000.

Piolino P, Belliard S, Desgranges B, Perron M, and Eustache F. Autobiographical memory and autonoetic consciousness in a case of semantic dementia. Cognitive Neuropsychology, 20: 619-639, 2003.

Quinette P, Guillery-Girard B, Dayan J, de la Sayette V, Marquis S, Viader F, Desgranges B, and Eustache F. What does transient global amnesia really mean? Review of the literature and thorough study of 142 cases. Brain, 129: 2508-2519, 2006.

Quinette P, Guillery B, Desgranges B, de la Sayette V, Viader F, and Eustache, F. Working memory and executive functions in transient global amnesia. Brain, 126: 1917-1934, 2003.

Spielberger CD. Manual for the State-Trait Anxiety. Consulting Psychologists Press, 1983. 
Stillhard G, Landis T, Schiess R, Regard M, and Sialer G. Bitemporal hypoperfusion in transient global amnesia: 99m-Tc-HM-PAO SPECT and neuropsychological findings during and after an attack. Journal of Neurology, Neurosurgery and Psychiatry, 53: 339$342,1990$.

Suzuki M, Fujii T, Tsukiura T, Okuda J, Umetsu A, Nagasaka T, Mugikura S, Yanagawa I, Takahashi S, and Yamadori A. Neural basis of temporal context memory: a functional MRI study. Neuroimage, 17: 1790-1796, 2002.

Tulving E. Memory and consciousness. Canadian Psychology, 26: 1-11, 1985.

Tulving E. Episodic memory: from mind to brain. Annual Review of Psychology, 53: 1-25, 2002.

Uttner I, Weber S, Freund W, Schmitz B, Ramspott M, and Huber R. Transient global amnesia--full recovery without persistent cognitive impairment. European Neurology; 58: 146-151, 2007.

Von Zerssen D, Koeller DM, and Rey ER. A scale for the objective evaluation of the state of subjective well-being as a method for longitudinal studies. Arzneimittelforschung, 20: 915-918, 1970.

Yonelinas AP. The nature of recollection and familiarity: A review of 30 years of research. Journal of Memory and Language, 46: 441-517, 2002.

Yonelinas AP and Jacoby LL. The relation between remembering and knowing as bases for recognition: Effects of size congruency. Journal of Memory and Language, 34: 622643, 1995. 